\title{
ON PARTICLE FILTERS FOR LANDMINE DETECTION USING IMPULSE GROUND PENETRATING RADAR
}

\author{
William Ng, Thomas C. T. Chan, H. C. So* \\ City University of Hong Kong \\ Hong Kong
}

\author{
K. C. Ho \\ University of Missouri-Columbia \\ Columbia, MO 65211, USA
}

\begin{abstract}
In this paper, we present an online stochastic approach for landmine detection based on ground penetrating radar (GPR) signals using sequential Monte Carlo (SMC) methods. Since the existence of true landmines is unknown and random, we propose to use the reversible jump Markov chain Monte Carlo (RJMCMC) in association with the SMC methods to jointly detect and localize landmines in the light of observations. Computer simulations on real GPR measurements demonstate the robust and consistent performance of the proposed method.
\end{abstract}

\section{INTRODUCTION}

Owing to the good penetration, depth resolution and excellent detection of metallic and nonmetallic objects, ground penetrating radar (GPR) has become an emerging technique for landmine detection [1-3]. A GPR system receives returned electromagnetic signal from the ground by which landmines can be located, if present. In reality, the signals originating from various types of ground surfaces, like soil or clay, are nearly indistinguishable from those of the genuine landmines. Thus robust and intelligent approaches for the problem are needed.

Typical landmine detection approaches are based on background removal and the corresponding techniques include adaptive background subtraction [4], background modelling using a timevarying linear prediction [5] and its improvement [6]. Zoubir et al. [7] have proposed to combine a Kalman filter (KF) for state estimation and a detection method based on statistic testing, but this approach heavily relies on the appropriate selection of parameters in order to perform properly. Tang et al. have suggested in [8] using sequential Monte Carlo (SMC) methods, also known as particle filtering (PF), [9-11] for landmine detection application, where the ground bounce signals are estimated and removed prior to localizing landmines.

In this paper, three major contributions are presented. Even though the data model adopted in this paper primarily follows that in [7], a simplified version is proposed with a smaller number of variables without sacrificing the overall localization performance. In a given scan of surface, not only is the number of objects unknown, but it is also varying. The second contribution is to employ the reversible jump Markov chain Monte Carlo (RJMCMC) [12] to perform a soft rather than a hard detection of possible landmines, followed by state estimation using PF. The last one is the evalua-

* The work described in this paper was supported by a grant from the Research Grants Council of the Hong Kong Special Administrative Region, China (Project No. CityU 119605) tion and demonstration of the superior and consistent performance of the proposed method using real GPR measurements [13].

The rest of the paper is organised as follows. In Section II, we provide the state-space model and the prior distribution and likelihood functions. In Section III we present the development of the SMC method with the RJMCMC for landmine detection application. Computer simulations and evaluations on real GPR data are included in Section IV, and conclusions are given in Section V.

\section{STATE-SPACE MODEL}

The radargram or B-scan of a GPR scan usually consists of $K$ distance measurements along the $x$-axis on every time in$\operatorname{dex} n \in\{1, \ldots, N\}$. Let $\phi_{l, k}=\left[\boldsymbol{\alpha}_{l, k}^{T}, \boldsymbol{\beta}_{l, k}^{T}\right]^{T}$ be a combined state vector containing the background and target vector signals, $\boldsymbol{\alpha}_{l, k} \in \mathcal{R}^{M \times 1}$ and $\boldsymbol{\beta}_{l, k} \in \mathcal{R}^{M \times 1}$, where $L=$ $\lfloor N / M\rfloor$ is the number of strips, $M$ is the strip size or successive times samples at a given distance index $k$, and ()$_{l, k}=$ $\left[()_{M l+1, k},()_{M l+2, k}, \ldots,()_{M l+M, k}\right]^{T}$. In addition, we define a stochastic existence variable $s_{l, k} \in\{0,1\}$, indicating whether or not within the current $M$ samples a landmine is present. Then a general state evolution model can be expressed as follows

$$
\phi_{l, k}=\boldsymbol{f}\left(\phi_{l, k-1}\right)+\boldsymbol{B}_{s_{l, k}} \boldsymbol{v}_{l, k},
$$

where $\boldsymbol{f}(\cdot)$ may be a linear or nonlinear dynamical function, $\boldsymbol{B}_{s_{l, k}}=\left[\begin{array}{cc}\boldsymbol{I}_{M} & \mathbf{0}_{M} \\ \mathbf{0}_{M} & s_{l, k} \times \boldsymbol{I}_{M}\end{array}\right]$ with $\boldsymbol{I}_{M}$ and $\mathbf{0}_{M}$ being an $M \times M$ identity and zero matrices. The vector $\boldsymbol{v}_{l, k} \in \mathcal{R}^{M \times 1}$ consists of zero-mean, white Gaussian random variables with covariance matrix $\boldsymbol{\Sigma}_{v}^{l}=\left[\begin{array}{cc}\sigma_{v, 0, l}^{2} \boldsymbol{I}_{M} & \mathbf{0}_{M} \\ \mathbf{0}_{M} & \sigma_{v, 1, l}^{2} \boldsymbol{I}_{M}\end{array}\right]$ for strip $l$.

When comparing the above model in (1) with that in [7], one may realize that there are two main differences. Firstly, the background signal $\boldsymbol{\alpha}_{l, k}$ is always present and varies in every scan $k$, regardless whether a target exists $\left(s_{l, k}=1\right)$ or not $\left(s_{l, k}=0\right)$. Secondly, the bias term suggested in the model in [7] has been combined with the target signal $\boldsymbol{\beta}_{l, k}$. The motivations of our changes are to simplfiy the dynamical model by reducing one unknown variable, and hence to improve the overall performance of target detection and localization.

For the random variable $s_{l, k}$, we model it by the stochastic relationship $s_{l, k}=s_{l, k-1}+\epsilon_{s}$ [14], where $\epsilon_{s}$ is an i.i.d. random variable such that its prior distribution function is given by

$$
p\left(s_{l, k} \mid s_{l, k-1}\right)=\left\{\begin{array}{l}
\operatorname{Pr}\left(\epsilon_{s}=-1\right)=p_{d}, \\
\operatorname{Pr}\left(\epsilon_{s}=0\right)=1-p_{b}-p_{d}, \\
\operatorname{Pr}\left(\epsilon_{s}=1\right)=p_{b},
\end{array}\right.
$$


where $p_{b}, p_{d} \in\{0,1\}$ are respectively the probabilities of incrementing and decrementing the number of targets such that $p_{b}=0$ if $s_{l, k-1}=1$ and $p_{d}=0$ if $s_{l, k-1}=0$.

From this point onwards, our parameters of interest are denoted by $\boldsymbol{\theta}_{l, k}=\left\{\phi_{l, k}, s_{l, k}\right\}$ for strip $l$, whose joint prior distribution function becomes

$$
\begin{aligned}
p\left(\boldsymbol{\theta}_{l, k} \mid \boldsymbol{\theta}_{l, k-1}\right) & =p\left(\phi_{l, k}, s_{l, k} \mid \phi_{l, k-1}, s_{l, k-1}\right), \\
& =p\left(\phi_{l, k} \mid \phi_{l, k-1}, s_{l, k}\right) \times p\left(s_{l, k} \mid s_{l, k-1}\right),
\end{aligned}
$$

where $p\left(\phi_{l, k} \mid \phi_{l, k-1}, s_{l, k-1}\right)$ and $p\left(s_{l, k} \mid s_{l, k-1}\right)$ are the prior distribution functions of $\phi_{l, k}$ and $s_{l, k}$, respectively.

It is further assumed that the states at different strips are statistically independent as considered in [7] such that the joint prior distribution function of $\boldsymbol{\theta}_{k}$ becomes

$$
p\left(\boldsymbol{\theta}_{k} \mid \boldsymbol{\theta}_{k-1}\right)=\prod_{l=0}^{L-1} p\left(\boldsymbol{\theta}_{l, k} \mid \boldsymbol{\theta}_{l, k-1}\right),
$$

where $\boldsymbol{\theta}_{k}=\left[\boldsymbol{\theta}_{0, k}, \ldots, \boldsymbol{\theta}_{L-1, k}\right]^{T}$ and $p\left(\boldsymbol{\theta}_{l, k} \mid \boldsymbol{\theta}_{l, k-1}\right)$ is the prior distribution function in (3).

For the observation model, we have $\boldsymbol{y}_{l, k}=\boldsymbol{g}\left(\boldsymbol{\theta}_{l, k}\right)+\boldsymbol{\epsilon}_{l, k}$, where the function $\boldsymbol{g}(\cdot)$ may be linear or nonlinear, and $\boldsymbol{\epsilon}_{l, k}$ is a zero-mean, white Gaussian random variable vector with covariance matrix $\boldsymbol{\Sigma}_{\epsilon_{l}}=\sigma_{\epsilon_{l}}^{2} \boldsymbol{I}_{M}$. As a result, the likelihood of the observation $\boldsymbol{y}_{l, k}$ due to $\boldsymbol{\theta}_{l, k}$ can be expressed as $p\left(\boldsymbol{y}_{l, k} \mid \boldsymbol{\theta}_{l, k}\right)=$ $\mathcal{N}\left(\boldsymbol{y}_{l, k} \mid \boldsymbol{g}\left(\boldsymbol{\theta}_{l, k}\right), \boldsymbol{\Sigma}_{\epsilon_{l}}\right)$.

Denoting $\boldsymbol{y}_{k}=\left[\boldsymbol{y}_{0, k}, \ldots, \boldsymbol{y}_{L-1, k}\right]^{T}$ and $\boldsymbol{\theta}_{k}=\left[\boldsymbol{\theta}_{0, k}, \ldots, \boldsymbol{\theta}_{L-1, k}\right]^{T}$ we would like to estimate $\boldsymbol{\theta}_{l, k}=\left\{\boldsymbol{\phi}_{l, k}, s_{l, k}\right\} \forall l$ sequentially upon the receipt of $\boldsymbol{y}_{k}$.

\section{SEQUENTIAL MONTE CARLO METHODS}

In the context of online parameter estimation using SMC methods or PFs, we are interested in approximating the posterior distribution $\pi\left(\boldsymbol{\theta}_{k} \mid \boldsymbol{y}_{1: k}\right)$ using $N_{s}$ number of Monte Carlo samples $\left\{\boldsymbol{\theta}_{k}^{(i)}\right\}$ for $i=\left\{1, \ldots, N_{s}\right\}$ with their associated importance weights [9-11,15-17]. These samples are propagated and updated using a sequential version of importance sampling as new measurements become available. Hence statistical inferences, such as expectation, maximum a posteriori (MAP) estimates, and minimum mean square error (MMSE), can be computed from these samples.

\section{III.1. Sequential Importance Sampling}

Since we have assumed that the states from different strips are statistically independent, we may separately rather than jointly compute the particles and their associated weights for every individual strip. In other words, we will have $L$ independent PFs, each of which estimates the posterior distribution function of $\boldsymbol{\theta}_{l, k}$ of a particular strip $l$. From a large set of $N_{s}$ particles $\left\{\boldsymbol{\theta}_{l, k-1}^{(i)}\right\}_{i=1}^{N_{s}}$ with their associated importance weights $\left\{w_{l, k-1}^{(i)}\right\}_{i=1}^{N_{s}}$, we approximate the posterior distribution function $\pi\left(\boldsymbol{\theta}_{l, k-1} \mid \boldsymbol{y}_{l, 1: k-1}\right)$ as $\pi\left(\boldsymbol{\theta}_{l, k-1} \mid \boldsymbol{y}_{l, 1: k-1}\right) \approx \sum_{i=1}^{N_{s}} w_{l, k-1}^{(i)} \delta\left(\boldsymbol{\theta}_{l, k-1}-\boldsymbol{\theta}_{l, k-1}^{(i)}\right)$, where $\delta(\cdot)$ is the Dirac delta function and every particle $\boldsymbol{\theta}_{l, k}^{(i)}$ is generated from $\boldsymbol{\theta}_{l, k}^{(i)} \sim q\left(\boldsymbol{\theta}_{l, k} \mid \boldsymbol{\theta}_{l, k-1}^{(i)}, \boldsymbol{y}_{l, 1: k}\right)$, for $i=\left\{1, \ldots, N_{s}\right\}$. Among many practical PFs, we choose to use the bootstrap PF [9] and assign $q\left(\boldsymbol{\theta}_{l, k} \mid \boldsymbol{\theta}_{l, k-1}^{(i)}, \boldsymbol{y}_{l, 1: k}\right)=p\left(\boldsymbol{\theta}_{l, k} \mid \boldsymbol{\theta}_{l, k-1}^{(i)}\right)$ as in (3). The associated importance weights $w_{l, k}^{(i)}$ can be recursively updated as follows

$$
w_{l, k}^{(i)} \propto w_{l, k-1}^{(i)} \times \frac{p\left(\boldsymbol{y}_{l, k} \mid \boldsymbol{\theta}_{l, k}^{(i)}\right) p\left(\boldsymbol{\theta}_{l, k}^{(i)} \mid \boldsymbol{\theta}_{l, k-1}^{(i)}\right)}{q\left(\boldsymbol{\theta}_{l, k}^{(i)} \mid \boldsymbol{\theta}_{l, k-1}^{(i)}, \boldsymbol{y}_{l, 1: k}\right)},
$$

with $\sum_{i=1}^{N_{s}} w_{l, k}^{(i)}=1$. It follows that the new set of particles $\left\{\boldsymbol{\theta}_{l, k}^{(i)}\right\}_{i=1}^{N_{s}}$ with the associated importance weights $\left\{w_{l, k}^{(i)}\right\}_{i=1}^{N_{s}}$ is then approximately distributed according to $\pi\left(\boldsymbol{\theta}_{l, k} \mid \boldsymbol{y}_{l, 1: k}\right)$.

Having the set of particles $\left\{\boldsymbol{\theta}_{l, k}^{(i)}, w_{l, k}^{(i)}\right\}$ for $i \in\left\{1, \ldots N_{s}\right\}$, we may compute the residual energies for all strips as $\boldsymbol{\eta}_{l, k}=$ $\sum_{i=1}^{N_{s}} w_{l, k}^{(i)} \times \operatorname{VAR}\left[\boldsymbol{y}_{l, k}-\boldsymbol{g}\left(\phi_{l, k}^{(i)}, 0\right)\right]$, where $\boldsymbol{g}\left(\phi_{l, k}^{(i)}, 0\right)$ is an estimate of observation $\boldsymbol{y}_{l, k}$ based solely on the background signals and $\operatorname{VAR}[\cdot]$ is the variance operator. That is, if the observation $\boldsymbol{y}_{l, k}$ contains background signal only, the residual energy $\boldsymbol{\eta}_{l, k}$ is expected to be a small value. On the other hand, if the observation $\boldsymbol{y}_{l, k}$ contains target signal, using the background signal alone to estimate $\boldsymbol{y}_{l, k}$ is going to yield a much larger value of $\boldsymbol{\eta}_{l, k}$. We also denote the aggregate residual energy signal by $\boldsymbol{\eta}_{k}=\sum_{l=1}^{L} \boldsymbol{\eta}_{l, k}$, where the residual energy signals are summed over all strips $l$ along the $x$-axis. This quantity will be used later when receiver operating characteristic (ROC) curves are prepared for performance evaluation in Section IV.

As the particle filter operates through time, only a few particles contribute significant importance weights in (5), leading to the well-known problem of degeneracy $[11,16]$. To avoid this, one needs to resample the particles according to their importance weights. That is, those particles with more significant weights will be selected more frequently than those with less significant weights. More detailed discussion of degeneracy and resampling can be found in [11]

\section{III.2. Reversible Jump Markov chain Monte Carlo}

Unlike some existing methods, like [7], rely on the computation of test statistics in an ad hoc fashion and compare it with some pre-determined threshold to deterministically decide if a landmine is present, we propose to devise the RJMCMC, a variation of Metropolis-Hastings (MH) algorithm $[18,19]$, to explore all possible model spaces and softly determine if a landmine is present in a given strip of the radargram.

In our application for a given strip $l$ at every $k$ and particle we randomly generate a new candidate $\boldsymbol{\theta}_{l, k}^{\star}=\left\{\boldsymbol{\theta}_{l, k}^{\star}, s_{l, k}^{\star}\right\}$ from a distribution function $d(\cdot \mid \cdot)$, which is conditional on $\boldsymbol{\theta}_{l, k}^{(i)}=$ $\left\{\boldsymbol{\theta}_{l, k}^{(i)}, s_{l, k}^{(i)}\right\}$. The candidate will be accepted with probability $\xi=\min \{1, r\}$, where

$$
r=\frac{\pi\left(\boldsymbol{\theta}_{l, k}^{\star} \mid \boldsymbol{y}\right) d\left(\boldsymbol{\theta}_{l, k}^{(i)} \mid \boldsymbol{\theta}_{l, k}^{\star}\right)}{\pi\left(\boldsymbol{\theta}_{l, k}^{(i)} \mid \boldsymbol{y}\right) d\left(\boldsymbol{\theta}_{l, k}^{\star} \mid \boldsymbol{\theta}_{l, k}^{(i)}\right)} \times \boldsymbol{J},
$$

with $\boldsymbol{J}$ being the Jacobian of the transformation from $\boldsymbol{\theta}_{l, k}^{(i)}$ to $\boldsymbol{\theta}_{l, k}^{\star}$. In effect, the proposed RJMCMC method jumps between parameter subspaces, thus visiting all relevant models. In particular three different moves are randomly selected to enable the exploration of the parameter subspace. For a given strip $l$ at distance index $k$,

1. For each particle $i=\left\{1, \ldots, N_{s}\right\}$ :

- Sample $u \sim U_{[0,1]}$.

- If $\left(u<p_{b}\right)$, then "birth move". 


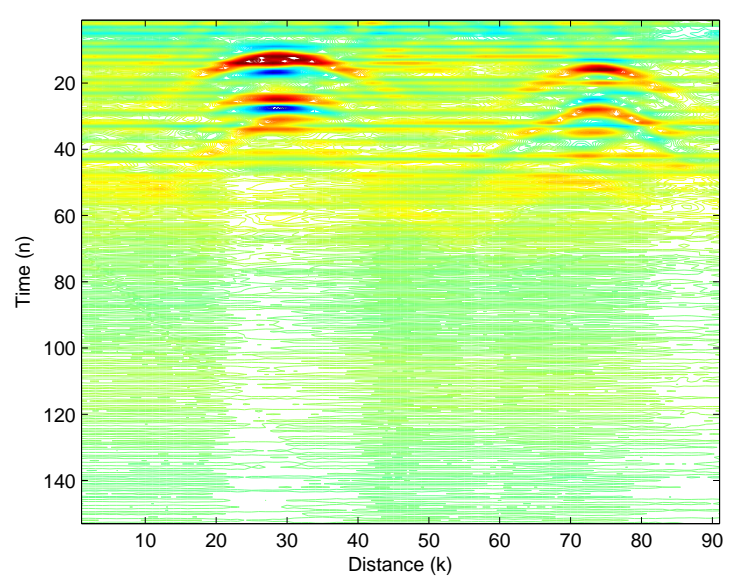

Fig. 1. The contour plot of the radargram from channel 46.

- Else, if $\left(u<p_{d}+p_{b}\right)$, then "death move".

- Else, update all parameters where $s_{l, k}=s_{l, k-1}$.

2. $k \leftarrow k+1$, goto step 1 .

In short, in the proposed method all $N_{s}$ particles assume different models according to the RJMCMC at a given scan $k$, and a histogram of the available models can be constructed from $\left\{s_{l, k}^{(i)}\right\}$ and a detection can be made if necessary.

\section{III.2.1. Birth, Death and Update Move}

When the birth move is selected, it is assumed that a landmine is present in the current region, i.e., $s_{l, k}=1$ given $s_{l, k-1}=0$. A candidate particle $\left\{\boldsymbol{\theta}_{l, k}^{\star}, s_{l, k}^{\star}=1\right\}$ is generated according to (3) and is accepted with probability $\xi_{\text {birth }}=\min \left\{1, r_{\text {birth }}\right\}$, where $r_{\text {birth }}=\exp \left\{-\frac{1}{2}\left(e^{\star^{T}} \boldsymbol{\Sigma}_{\epsilon}^{-1} e^{\star}-e^{\prime T} \boldsymbol{\Sigma}_{\epsilon}^{-1} e^{\prime T}\right)\right\}$, where $\boldsymbol{e}^{\star}=\boldsymbol{y}_{l, k}-\boldsymbol{g}\left(\boldsymbol{\theta}_{l, k}^{\star}\right)$ and $\boldsymbol{e}^{\prime}=\boldsymbol{y}_{l, k}-\boldsymbol{g}\left(\boldsymbol{\theta}_{l, k}^{\prime}\right)$, and $\boldsymbol{\theta}_{l, k}^{\prime} \sim$ $p\left(\boldsymbol{\theta}_{l, k} \mid \boldsymbol{\theta}_{l, k-1}, s_{l, k}^{\prime}=0, s_{l, k-1}=0\right)$. When the death move is selected, similar procedures in the birth move are taken in which a candidate particle $\boldsymbol{\theta}_{l, k}^{\star}$ with $s_{l, k}^{(i)}=0$ and another particle $\boldsymbol{\theta}_{l, k}^{\prime}$ with $s_{l, k}^{(i)}=1$ are generated. The candidate particle will be accepted with probability $\xi_{\text {death }}=\min \left\{1, \frac{1}{r_{\text {birth }}}\right\}$. The particle $\boldsymbol{\theta}_{l, k}^{(i)}=\left\{\boldsymbol{\theta}_{l, k}^{(i)}, s_{l, k}^{(i)}\right\}$, where $\left\{\boldsymbol{\theta}_{l, k}^{(i)}, s_{l, k}^{(i)}\right\}=\left\{\boldsymbol{\theta}_{l, k}^{\star}, s_{l, k}^{\star}\right\}$ if the candidate is accepted. Otherwise, $\left\{\boldsymbol{\theta}_{l, k}^{(i)}, s_{l, k}^{(i)}\right\}=\left\{\boldsymbol{\theta}_{l, k}^{\prime}, s_{l, k}^{\prime}\right\}$. The new particle $\boldsymbol{\theta}_{l, k}^{(i)}$ will then be used to update the importance weights $w_{l, k}^{(i)}$. If, however, the update move is selected, we simply generate the particle $\boldsymbol{\theta}_{l, k}^{(i)} \sim q\left(\boldsymbol{\theta}_{l, k} \mid \boldsymbol{\theta}_{l, k-1}^{(i)}, \boldsymbol{y}_{l, 1: k}\right)$ with $s_{l, k}^{(i)}=s_{l, k-1}^{(i)}$, which will then be used to compute the importance weights $w_{l, k}^{(i)}$.

\section{SIMULATION RESULTS}

In this section the performance of the proposed algorithm on a set of real GPR measurements [13] is evaluated. Furthermore, the results from the proposed method will be compared with those from

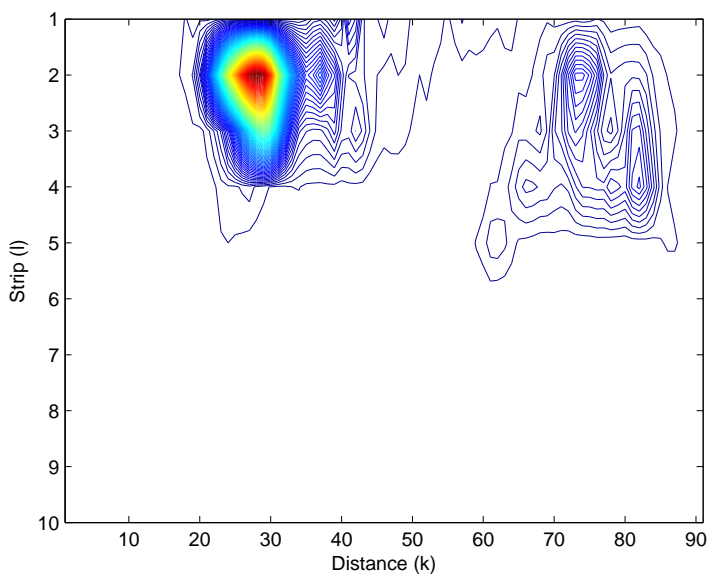

Fig. 2. The contour plot of localization results of the radargram in Fig. 1 from the proposed method averaged over 100 independent trials.

the Zoubir's method in [7]. Here we specify the form of the dynamical function $f(\cdot)$ and observation function $g(\cdot)$. In particular, we choose to use the same linear model as in [7] in order to carry out a fair performance comparison. Accordingly, the dynamical function is $\boldsymbol{f}\left(\phi_{l, k-1}\right)=\boldsymbol{I}_{2 M} \boldsymbol{\phi}_{l, k-1}$ and the observation function becomes $\boldsymbol{g}_{j}\left(\boldsymbol{\theta}_{l, k}\right)=\boldsymbol{H}_{j}=\left[\begin{array}{ll}\boldsymbol{I}_{M} & j \times \boldsymbol{I}_{M}\end{array}\right] \boldsymbol{\phi}_{l, k}$, for $j=\{0,1\}$. Note that for other forms of functions, say nonlinearity, chosen for $\boldsymbol{f}(\cdot)$ and $\boldsymbol{g}(\cdot)$ would not drastically affect the performance of the proposed SMC algorithms as they are developed to tackle problems that are nonlinear and non-Gaussian [9-11,15-17].

In the data set [13] there are a total of 91 channels, and in this evaluation we consider one sub-set of channels from $42-49$, where there are two landmine objects centered at the range of $x$ positions: $28-29 \mathrm{~cm}$ and $72-75 \mathrm{~cm}$, respectively. Fig. 1 exhibits the radargram with $K=91$ distance measurements and $N=150$ collected in one of the channels, where the first 50 rows of data have been taken away as the signals in this block correspond to the responses to the ground surface which are inappropriate for localization purposes.

The proposed method is investigated in this experiment for localizing the objects with $M=10, N_{s}=500$, and $p_{d}=p_{b}=0.2$. A total of 100 independent trials are conducted on these channels. According to the contour plots in Fig. 2, the proposed method clearly localizes the objects when compared with the true radagrams in Fig. 1.

To quantitatively evaluate the localization performance on the real measurements, the ROC curves are constructed for the results from the proposed method. Prior to constructing ROC curves for the evaluation on both methods, we need to first define the assumed width of a genuine landmine. In the absence of this piece of information, we assume the width of a landmine is 7 distance indicies, i.e., 3 indicies left and right to its assumed center. For comparison purposes the Zoubir's approach is also studied with these sets of measurements. From Fig. 3, it is seen that the proposed method outperforms the Zoubir's approach when real measurements are considered.

In summary according to the ROC curves the proposed ap- 


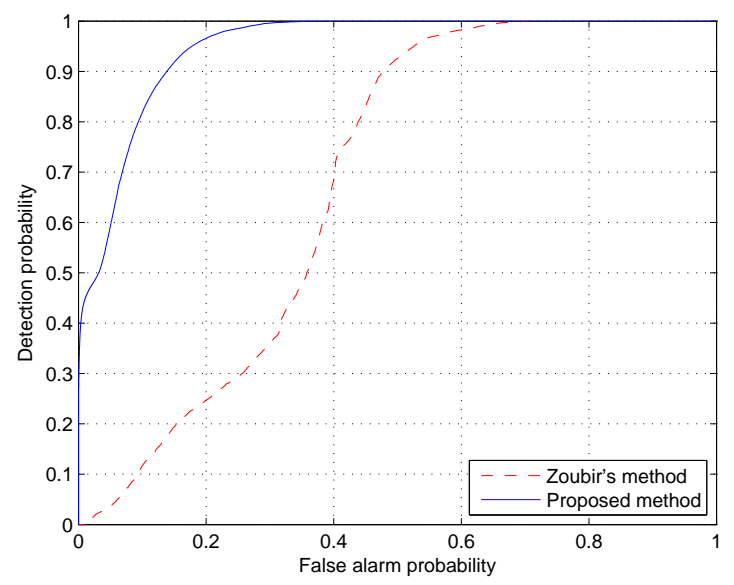

Fig. 3. The ROC curves obtained from the proposed and the Zoubir's methods on the data sets over 100 independent trials.

proach is able to consistently localize the landmine objects from real GPR data using the PF and RJMCMC method, and is superior to the Zoubir's method. Nevertheless, it requires higher computational load when compared with that of the Zoubir's and other competing non-PF based methods.

\section{CONCLUSION}

A stochastic online landmine detection method for ground penetrating radar data using the sequential Monte Carlo approach with reversible jump Markov chain Monte Carlo (RJMCMC) has been presented. The proposed method in association with the simplied Zoubir's data model takes the advantage of the RJMCMC to explore different model spaces and to expend the extensive computation only on the most possible model space. The benefit is obvious that no hard or predetermined thresholds are needed to decide which model should be used given the data. Computer simulations demonstrate that the proposed approach is able to successfully localize the landmine objects from real GPR data, and it outperforms the Zoubir's method at the expense of higher computational load.

\section{REFERENCES}

[1] L. Peter Jr., J. J. Daniel, and J. D. Young, "Ground penetrating radar as a subsurface environmental sensing tool," Proceedings of the IEEE, vol. 82, pp. 1802-1822, Dec. 1994.

[2] D. J. Daniels, Surface-Penetrating Radar, Institution of Electrical Engineers, London, 1996.

[3] T. R. Witten, "Present state of the art in ground-penetrating radars for mine detection," in Proc. SPIE Conf., Orlando, FL, 1998, vol. 3392, pp. 576-586.

[4] R. Wu, A. Clement, J. Li, E. G. Larsson, M. Bradley, J. Habersat, and G. Maksymonko, "Adaptive ground bounce removal," Electronics Letters, vol. 37, no. 20, pp. 12501252, 2001.

[5] K. C. Ho and P. D. Gader, "A linear prediction land mine detection algorithm for hand held ground penetrating radar,"
IEEE Transactions on Geosci. Remote Sensing, vol. 40, no. 6, pp. 1374-1384, 2002.

[6] K. C. Ho, P. D. Gader, and J. N. Wilson, "Improving landmine detection using frequency domain features from ground penetrating radar," in Proc. IEEE International Conference on Geosci. Remote Sensing Symposium, 2004, vol. 3, pp. 1617-1620.

[7] A. M. Zoubir, I. J. Chant, C. L. Brown, B. Barkat, and C. Abeynayake, "Signal processing techniques for landmine detection using impulse ground penetrating radar," IEEE Sensors Journal, vol. 2, no. 1, pp. 41-51, 2002.

[8] L. Tang, P. A. Torrione, C. Eldeniz, and L. M. Collins, "Ground bounce tracking for landmine detection using a sequential Monte Carlo method," in Proceedings of SPIE, 2007, vol. 6553.

[9] N.J. Gordon, D.J. Salmond, and A.F.M. Smith, "Novel approach to non-linear/non-Gaussian Bayesian state estimation," IEE Proceedings- $F$, vol. 140, no. 2, pp. 107-113, 1993.

[10] G. Kitagawa, "Monte Carlo filter and smoother for nonGaussian nonlinear state space models," Journal of Computational and Graphical Statistics, vol. 5, no. 1, pp. 1-25, 1996.

[11] A. Doucet, S. Godsill, and C. Andrieu, "On sequential Monte Carlo sampling methods for Bayesian filtering," Statistics and Computing, vol. 10, pp. 197-208, 2000.

[12] P.J. Green, "Reversible jump Markov chain Monte Carlo computation and Bayesian model determination," Biometrika, vol. 82, no. 4, pp. 711-732, 1995.

[13] ," http://users.ece.gatech.edu/ wrscott/.

[14] J. R. Larocque, J. P. Reilly, and W. Ng, "Particle filter for tracking an unknown number of sources," IEEE Transactions on Signal Processing, vol. 50, no. 12, pp. 2926-2937, Dec. 2002.

[15] O. Cappé, S. J. Godsill, and E. Moulines, "An overview of existing methods and recent advances in sequential Monte Carlo," in IEEE Transactions on Large Scale Dynamical Systems Workshop, 2007, vol. 95, pp. 889-924.

[16] A. Doucet, N. de Freitas, and N. Gordon, Eds., Sequential Monte Carlo in Practice, Springer-Verlag, New York, 2001.

[17] J. Liu and R. Chen, "Sequential Monte Carlo methods for dynamic systems," Journal of the American Statistical Association, vol. 93, no. 443, pp. 1032-1044, 1993.

[18] J. Bernardo and A. Smith, Bayesian Theory, Wiley \& Sons Ltd., New York, 1994.

[19] W.K. Hastings, "Monte Carlo sampling methods using Markov chains and their applications," Biometrika, vol. 57, no. 1, pp. 97-109, 1970. 